\title{
Role of hyperfine interaction for cavity-mediated coupling between spin qubits
}

\author{
Julia Hildmann and Guido Burkard \\ Department of Physics, University of Konstanz, D-78457 Konstanz, Germany
}

\begin{abstract}
We consider two qubits interacting by means of an optical cavity, where each qubit is represented by a single electron spin confined to a quantum dot. It is known that electron spins in III-V semiconductor quantum dots are affected by the decoherence due to the hyperfine interaction with nuclear spins. Here we show that the interaction between two qubits is influenced by the Overhauser field as well. Starting from an unpolarizied nuclear ensemble, we investigate the dependance of the fidelities for two-qubit gates on the Overhauser field. We include the hyperfine interaction perturbatively to second order in our analytical results, and to arbitrary precision numerically.
\end{abstract}

\section{INTRODUCTION}

Substantial progress has been made towards the implementation of coherent interfaces between an electron spin in a quantum dot and photons [1 4]. The coupling of single spins to photons is a promising mechanism for implementing quantum information processing schemes based on quantum dots, where a qubit (quantum bit of information) is represented by electron spin degrees of freedom [5]. The main source of electron spin decoherence in semiconductor quantum dots is considered to be the hyperfine interaction to the nuclear spins of the lattice atoms [6, 7]. The dephasing time due to the interaction with an unprepared ensemble of nuclear spins is about 10 ns [7, 8], which is much shorter than the single qubit operation times by means of electronic control $(\approx 100 \mathrm{~ns}$ [9]). There are some ways to deal with this problem: to prolong the decoherence time using advanced spin-echo techniques [10] or dynamical nuclear polarization [11, to use nuclear spin "poor" materials such as graphene [12] or nitrogen-vacancy centers in diamond 13 or to shorten the qubit manipulation time [14. The optical driving of electron spins has this last advantage and offers mechanisms for single qubit operation time around 30 ps [2]. Besides single qubit manipulations, two qubit operations are required for implementing quantum algorithms. One of the schemes for optical exchange interaction between two spins is based on virtual Raman transitions between valence band heavy holes and conduction band electrons [15]. Thereby, a set of quantum dots is coupled to a high finesse cavity. Each quantum dot is doped with a single electron and can be controlled by linearly polarized laser light. The laser polarization is perpendicular to the growth direction of the quantum dots and perpendicular to the cavity field polarization as well, so that laser light is acting on the quantum dots without driving the cavity. A magnetic field is applied in the Voigt configuration, i.e., perpendicular to the growth direction and parallel to the cavity field polarization, so that single photons emited by quantum dots escape into the cavity and can be detected afterwards. The cavity frequency is chosen such that it is slightly detuned from the transition from the electron spin down state to the trion state, while the laser frequency is detuned from the transition from the spin up state to the trion state. If these detun-

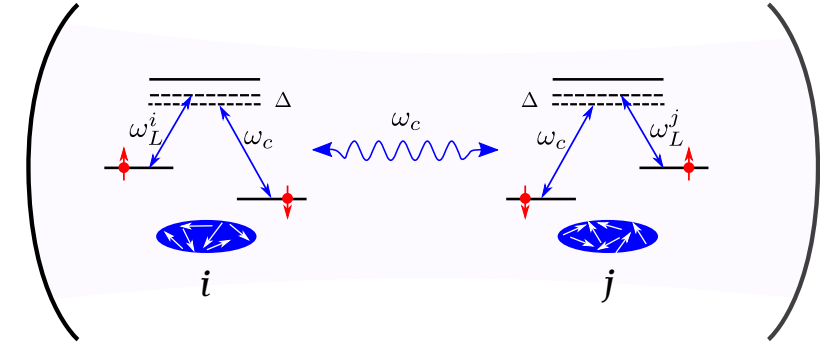

Figure 1. Cavity mode mediated spin-spin interaction. If the difference of the detunings $\Delta$ for two quantum dots coincides, then they can exchange virtual cavity photons of energy $\omega_{c}$. Here, $\omega_{c}$ and $\omega_{L}^{i}$ are the frequencies of the cavity mode and the laser acting on quantum dot $i$, and $\Delta$ denotes the difference between the detunings, i.e. the detuning from the two-photon resonance on a given quantum dot.

ings are the same then the $\Lambda$ system is in a two-photon resonance that allows for manipulating a single electron spin (Figs. 1 and 2). If the detunings in a quantum dot are different, but the difference of the detunings (the detuning from the two-photon resonance) match for a pair of quantum dots, then the electron spins in these quantum dots can resonantly exchange energy through virtual cavity photons (Fig. 1). In this way, virtual photons can be used for performing coherent interaction between arbitrary qubits. Two-qubit operations can potentially be carried out on a subnanosecond timescale [15], therefore the decoherence can be expected to originate mainly from nuclear spins. In this paper, we calculate the fidelities of such two-qubit operations in the presence of the hyperfine interaction. We will study in particular partial SWAP operations and the controlled-not (CNOT) gate. For this purpose, we first calculate the fidelity of the quantum gates of interest analytically to second order of the Overhauser field and subsequently we perform an exact numerical calculation for comparison.

\section{MODEL}

We start from the microscopic model describing a quantum dot embedded in a cavity and irradiated by a 
laser field,

$$
\begin{aligned}
H= & \sum_{\sigma=\uparrow, \downarrow, \pm 3 / 2} \omega_{\sigma} e_{\sigma}^{\dagger} e_{\sigma}+\omega_{c} a_{c}^{\dagger} a_{c}+\omega_{L} a_{L}^{\dagger} a_{L}+H_{\mathrm{hf}} \\
& +g_{c} a_{c}^{\dagger} e_{v}^{\dagger} e_{\uparrow}-i g_{L} a_{L}^{\dagger} e_{v}^{\dagger} e_{\downarrow}+h . c .
\end{aligned}
$$

where $\omega_{\sigma}$ and $e_{\sigma}$ are the energy and annihilation operators for the electronic states in the conduction band with spin $\sigma= \pm 1 / 2=\uparrow, \downarrow$ and in the valence band with total angular momentum $\sigma= \pm 3 / 2$ (we assume that the heavy hole subband is sufficiently split from the light hole band to allow for pure heavy hole excitation). The quantization axis for the electrons is set by the external magnetic field along the $x$ direction, while the quantization axis in the valence band is given by the structure and assumed to be in $z$ direction. Furthermore, for a linearly polarized cavity mode $\left(\omega_{c}, a_{c}\right)$ along the $x$ direction and linearly polarized laser mode $\left(\omega_{L}, a_{L}\right)$ in the $y$ direction, we obtain a radiative coupling to the linear combination $e_{v}=\left(e_{-3 / 2}-e_{3 / 2}\right) / \sqrt{2}$ in the valence band. The coupling strengths to the cavity and laser modes are denoted with $g_{c}$ and $g_{L}$, respectively. The hyperfine interaction of a conduction band electron in the quantum dot with the surrounding nuclear spins can be written as

$$
H_{\mathrm{hf}}=\mathbf{S} \cdot \sum_{k=1}^{N} A_{k} \mathbf{I}^{k},
$$

where $\mathbf{I}^{k}$ is the operator belonging to the $k$-th nuclear spin in contact with the electron and $A_{k}$ denotes the corresponding hyperfine coupling constant. The average hyperfine coupling constant for GaAs is $90 \mu \mathrm{eV}$ [16. The electron spin operator is given by $\mathbf{S}=$ $\frac{1}{2} \sum_{\sigma, \sigma^{\prime}=\uparrow, \downarrow} e_{\sigma}^{\dagger} \boldsymbol{\sigma}_{\sigma \sigma^{\prime}} e_{\sigma^{\prime}}$, where $\boldsymbol{\sigma}=\left(\sigma_{x}, \sigma_{y}, \sigma_{z}\right)$ is the vector consisting of Pauli matrices. Here, we neglect the dipolar hyperfine coupling of the valence band states since it is typically smaller. In the presence of a magnetic field along the $x$ direction exceeding the nuclear field of typically $\sim 10 \mathrm{mT}$, we can neglect the flip-flop terms and write $H_{\mathrm{hf}} \approx S_{x} \sum_{k=1}^{N} A_{k} I_{x}^{k} \equiv S_{x} h$, where $h$ denotes the Overhauser (nuclear) field operator in $x$ direction. We do not take the nuclear Zeeman terms into account, because they are considerably smaller than the thermal energy and the electron Zeeman energy. We can then combine the hyperfine Hamiltonian with the first term in Eq. (1) by using $\omega_{\sigma}=\left(g_{e} \mu_{B} B+h\right) \sigma / 2$ for $\sigma=\uparrow, \downarrow= \pm 1$, where $B$ is the magnetic field applied along $x$ and $g_{e}$ is the effective electron g-factor. We can now replace the operator $h$ with one of its eigenvalues and perform an average over $h$ later. This allows us to follow the steps performed in Ref. [15] before taking the average over nuclear configurations. In GaAs quantum dots the number $N$ of nuclear spins is large, typically between $10^{5}$ and $10^{6}$, and therefore the Overhauser field follows a Gaussian distribution around mean value 0 and with variance $\sigma \simeq A / \sqrt{N}$ [16].

In order to eliminate the valence band states, we perform a Schrieffer-Wolff transformation $H_{\text {eff }}=e^{-S} H e^{S}$ with the anti-Hermitian operator

$$
S=\frac{g_{c}}{\omega_{\uparrow}-\omega_{v}-\omega_{c}} a_{c}^{\dagger} e_{v}^{\dagger} e_{\uparrow}-i \frac{g_{L}}{\omega_{\downarrow}-\omega_{v}-\omega_{L}} a_{L}^{\dagger} e_{v}^{\dagger} e_{\downarrow}-h . c .,
$$

where we use $\omega_{3 / 2} \approx \omega_{-3 / 2} \equiv \omega_{v}$. By setting the valence band occupation number to $e_{v}^{\dagger} e_{v} \approx 1$ and using that the laser field is in a coherent state with $g_{L} a_{L} \approx$ $\Omega_{L} \exp \left(-i \omega_{L} t\right)$, we obtain to second order in the cavity and laser couplings $g_{c}$ and $g_{L}$,

$$
\begin{aligned}
H_{\mathrm{eff}}=\omega_{c} a_{c}^{\dagger} a_{c}+ & \sum_{i=1,2}\left[\left(g_{e} \mu_{B} B+h_{i}\right) \sigma_{\uparrow \uparrow}^{i}\right. \\
& +i g_{\mathrm{eff}}^{i}\left(a_{c}^{\dagger} \sigma_{\downarrow \uparrow}^{i} e^{-i \omega_{L}^{i} t}-h . c .\right) \\
& \left.-\frac{g_{c}^{2}}{\Delta_{c}^{i}\left(h_{i}\right)} \sigma_{\downarrow \downarrow}^{i} a_{c}^{\dagger} a_{c}-\frac{\left(\Omega_{L}^{i}\right)^{2}}{\Delta_{L}^{i}\left(h_{i}\right)} \sigma_{\uparrow \uparrow}^{i}\right],
\end{aligned}
$$

where the index $i$ refers to the respective quantities in $\operatorname{dot} i=1,2$, and $\sigma_{i j}=|i\rangle\langle j|, i, j=\uparrow, \downarrow$. The first term inside the sum in Eq. (4) is the Zeeman splitting due to the external and nuclear fields, the second term describes the effective coupling of the cavity mode to the quantum dot electron spins with strength

$$
g_{\mathrm{eff}}^{i}(t)=\frac{g_{c} \Omega_{L}^{i}(t)}{2}\left(\frac{1}{\Delta_{c}^{i}\left(h_{i}\right)}+\frac{1}{\Delta_{L}^{i}\left(h_{i}\right)}\right),
$$

where

$$
\begin{aligned}
\Delta_{c}^{i}\left(h_{i}\right) & =\frac{g_{e} \mu_{B} B+h_{i}}{2}-\omega_{v}-\omega_{c}=\Delta_{c}^{i}+\frac{h_{i}}{2}, \\
\Delta_{L}^{i}\left(h_{i}\right) & =-\frac{g_{e} \mu_{B} B+h_{i}}{2}-\omega_{v}-\omega_{L}^{i}=\Delta_{L}^{i}-\frac{h_{i}}{2},
\end{aligned}
$$

are the detunings of the cavity and laser fields (s. Fig. 2), and the last two terms in Eq. (4) can be interpreted as the Lamb and Stark shifts of the cavity and quantum dot levels, respectively.

A second Schrieffer-Wolff transform can be used to also eliminate the cavity mode, which leads to the effective photon-mediated interaction between two spins $i$ and $j$ in the interaction picture with

$H_{0}=\sum_{i}\left(g_{e} \mu_{B} B+h_{i}\right) \sigma_{\uparrow \uparrow}^{i}$,

$$
H_{\mathrm{int}}^{i j}=\frac{\tilde{g}_{i j}(t)}{2}\left(\sigma_{\uparrow \downarrow}^{i} \sigma_{\downarrow \uparrow}^{j} \mathrm{e}^{i\left(h_{i}-h_{j}\right) t}+\sigma_{\uparrow \downarrow}^{j} \sigma_{\downarrow \uparrow}^{i} \mathrm{e}^{-i\left(h_{i}-h_{j}\right) t}\right),
$$

where

$$
\tilde{g}_{i j}(t)=\frac{g_{\mathrm{eff}}^{i}(t) g_{\mathrm{eff}}^{j}(t)}{2}\left(\frac{1}{\Delta_{i}\left(h_{i}\right)}+\frac{1}{\Delta_{j}\left(h_{j}\right)}\right),
$$

with

$$
\Delta_{i}\left(h_{i}\right)=\Delta_{c}^{i}\left(h_{i}\right)-\Delta_{L}^{i}\left(h_{i}\right)=\Delta_{i}+h_{i},
$$

represents the coupling strength between two electron spins. The time-independent interaction Hamiltonian Eq. (8) strictly applies to the two-photon resonance 


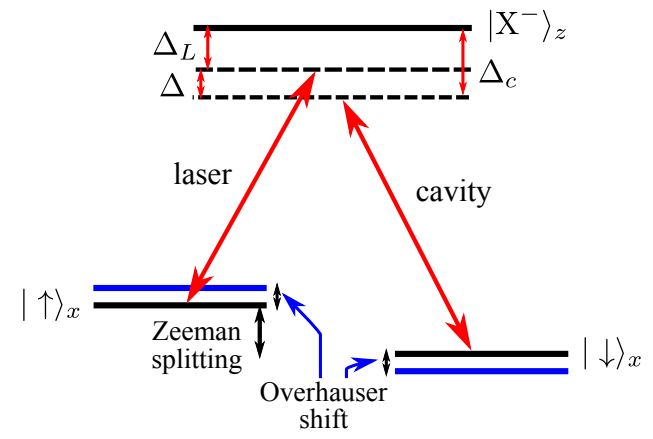

Figure 2. Energy level scheme for a quantum dot filled with a single electron and coupled to a cavity mode. The Zeemansplit single-electron states can be excited to a trion (negatively charged exciton) state $\left|X^{-}\right\rangle$by coupling to the cavity or laser field. Both cavity and laser field frequencies are detuned by $\Delta_{c}$ and $\Delta_{L}$ from resonance, and the combined system is detuned from its two-photon resonance by $\Delta=\Delta_{c}-\Delta_{L}$. The Overhauser shift caused by the hyperfine coupling to the nuclear spins leads to a fluctuating detuning, thus reducing the fidelity of the optically generated quantum gates.

$\Delta_{i}=\Delta_{j} \equiv \Delta$ in the absence of nuclear spins. By going over into the rotating frame with nuclear spins by $R=$ $\exp \left(i t\left(h_{i} \sigma_{\uparrow \uparrow}^{i}+h_{j} \sigma_{\uparrow \uparrow}^{j}\right)\right)$ we obtain a time-independent effective interaction Hamiltonian with Heisenberg transverse coupling type between two electron spins:

$$
\tilde{H}_{i j}^{\mathrm{int}}=R^{\dagger} H_{\mathrm{int}}^{i j} R=\frac{\tilde{g}_{i j}(t)}{2}\left(\sigma_{y}^{i} \sigma_{y}^{j}+\sigma_{z}^{i} \sigma_{z}^{j}\right) .
$$

The unitary time evolution operator of the interaction between two electron spins is

$$
\tilde{U}(\phi)=\exp \left[-i \int \tilde{H}_{\mathrm{int}}^{i j} d t\right]
$$

with $\phi=\int \tilde{g}_{i j}(t) d t$. The time evolution operator in original frame is then

$$
\begin{aligned}
U(\phi) & =R \tilde{U}(\phi) R^{\dagger}= \\
& =\left(\begin{array}{cccc}
1 & 0 & 0 & 0 \\
0 & \cos (\phi) & i \sin (\phi) \mathrm{e}^{i\left(h_{i}-h_{j}\right) t} & 0 \\
0 & i \sin (\phi) \mathrm{e}^{-i\left(h_{i}-h_{j}\right) t} & \cos (\phi) & 0 \\
0 & 0 & 0 & 1
\end{array}\right) .
\end{aligned}
$$

In the subspace $\{|\uparrow \downarrow\rangle,|\downarrow \uparrow\rangle\}$ the operator $U(\phi)$ acts as a rotation $\exp (i \phi \boldsymbol{\sigma} \cdot \hat{n})$ with $\hat{n}=\left(-\cos \left\{\left(h_{i}-\right.\right.\right.$ $\left.\left.\left.h_{j}\right) t\right\}, \sin \left\{\left(h_{i}-h_{j}\right) t\right\}, 0\right)$. Thus the changed time evolution of the two-electron spin state due to hyperfine interaction can be interpreted as a modified rotation in the mentioned subspace (s. Fig. 3). There are two distinct effects due to the nuclear spins. First, the interaction phase becomes Overhauser field dependent $\phi=\phi\left(h_{i}, h_{j}\right)$ and, secondly, the rotation axis starts to precess in the x-y plane with $\left(h_{i}-h_{j}\right) t$. The second effect has the maximal contribution when $\phi(0)=m \frac{\pi}{2}$, where $\phi(0)=\tilde{g}_{i j}\left(h_{i}=0, h_{j}=0\right) t \equiv \tilde{g}_{i j}(0) t$ and where $m$ is an integer. When $\phi(0)=m \pi$, the trajectory of the

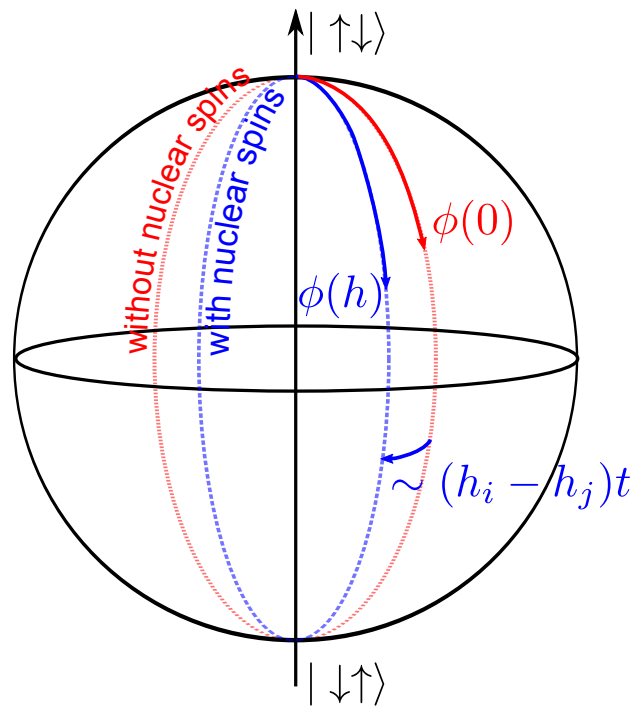

Figure 3. Effect of the Overhauser fields $h_{i}$ and $h_{j}$ on the time evolution of the two-electron spin state: change of the interaction phase and precessing of the rotation axis in the x-y plane with $\left(h_{i}-h_{j}\right) t$

two-electron spin state affected by nuclear spins coincides with an unaffected one.

The two-qubit CNOT operation can be implemented as a sequence of single spin rotations combined with the unitary time evolution operator [15]:

$$
\begin{aligned}
& U_{\text {CNOT }}=\mathrm{e}^{-i \frac{\pi}{4} \sigma_{z}^{j}} \mathrm{e}^{-i \frac{\pi}{4}} \mathrm{e}^{-i \frac{\pi}{3} \hat{n}_{i} \cdot \sigma_{i}} \mathrm{e}^{-i \frac{\pi}{3} \hat{n}_{j} \cdot \sigma_{j}} \times \\
& \times U\left(\frac{\pi}{4}\right) \mathrm{e}^{-i \frac{\pi}{2} \sigma_{z}^{i}} U\left(\frac{\pi}{4}\right) \mathrm{e}^{-i \frac{\pi}{4} \sigma_{y}^{i}} \mathrm{e}^{-i \frac{\pi}{4} \sigma_{y}^{j}} \mathrm{e}^{i \frac{\pi}{4} \sigma_{z}^{j}},
\end{aligned}
$$

where $\hat{n}_{i}=(1,1,-1) / \sqrt{3}, \hat{n}_{j}=(-1,1,1) / \sqrt{3}$.

We calculate the fidelity of the generated unitary gate in the presence of the Overhauser field with respect to the ideal unitary gate (without Overhauser field) in order to quantify the effect of the nuclear spins. This fidelity reflects the difference in final state after the gate operation, averaged over pure input quantum states [17. The average fidelity for operators acting in 4-dimensional Hilbert space is given by [18]

$$
F(\hat{O}(0), \hat{O}(h))=\frac{4+\left|\operatorname{Tr}\left(\hat{O}^{\dagger}(0) \hat{O}(h)\right)\right|^{2}}{20},
$$

where $\hat{O}(h)$ and $\hat{O}(0)$ denote the unitary operator with and without Overhauser field dependence. Next, the average fidelity Eq. 15 must be averaged over the nuclear spin field distributions in the quantum dots $i$ and $j$ to extract the Overhauser field dependence of the fidelities for the time evolution and the CNOT operator. 


\section{FIDELITIES IN SECOND ORDER HYPERFINE INTERACTION}

Starting from the microscopic model Eq. (1) and performing two subsequent Schrieffer-Wolff transformations [15] while including the hyperfine interaction, we find the effective spin-spin interaction Hamiltonian as described above. Using Eqs. (5), (6), (7), (9), and (10), we find that the effective spin-spin coupling depends on the Overhauser fields $h_{i}$ and $h_{j}$ in the quantum dots,

$$
\begin{aligned}
\tilde{g}_{i j}\left(h_{i}, h_{j}\right)= & \frac{g_{c}^{2} \Omega_{L}^{i} \Omega_{L}^{j}}{16}\left(\frac{1}{\Delta_{i}+h_{i}}+\frac{1}{\Delta_{j}+h_{j}}\right) \\
& \times\left(\frac{1}{\Delta_{c}^{i}+h_{i} / 2}+\frac{1}{\Delta_{L}^{i}-h_{i} / 2}\right) \\
& \times\left(\frac{1}{\Delta_{c}^{j}+h_{j} / 2}+\frac{1}{\Delta_{L}^{j}-h_{j} / 2}\right) .
\end{aligned}
$$

We assume that the fluctuations of the Overhauser field around its zero mean value are small, since $h \sim A \sim 10^{-5}$ $\mathrm{eV}$. Therefore we can investigate analytically the fidelities to the second order of the Overhauser field. Since the interaction Hamiltonian (8) is nuclear field dependent partly through the coupling coefficient $\tilde{g}_{i j}$, we need to expand it with respect to the Overhauser field in both coupled quantum dots,

$$
\begin{aligned}
\frac{\tilde{g}_{i j}\left(h_{i}, h_{j}\right)}{\tilde{g}_{i j}(0)}= & 1+\frac{h_{i} h_{j}}{4}\left(\frac{\Delta^{2}}{\Delta_{c}^{i} \Delta_{L}^{i} \Delta_{c}^{j} \Delta_{L}^{j}}-\frac{1}{\Delta_{c}^{i} \Delta_{L}^{i}}-\frac{1}{\Delta_{c}^{j} \Delta_{L}^{j}}\right) \\
& +\sum_{l=i, j}\left[\frac{h_{l}}{2}\left(\frac{\Delta}{\Delta_{c}^{l} \Delta_{L}^{l}}-\frac{1}{\Delta}\right)\right. \\
& \left.+\frac{h_{l}^{2}}{4}\left(\frac{\Delta^{2}}{\Delta_{c}^{l 2} \Delta_{L}^{l 2}}+\frac{2}{\Delta^{2}}\right)\right]+\mathcal{O}\left(h^{3}\right) .
\end{aligned}
$$

The trace of the product of the perfect time evolution operator and the one with included hyperfine interaction is $\operatorname{Tr}\left[U^{\dagger}(0) U(h)\right]=2\left\{1+\cos [\phi(0)] \cos (\phi)+\cos \left[\left(h_{i}-\right.\right.\right.$ $\left.\left.\left.h_{j}\right) t\right] \sin [\phi(0)] \sin (\phi)\right\}$. We obtain for the average fidelity between those two operators to the second order of the Overhauser fields $h_{i}$ and $h_{j}$,

$$
\begin{aligned}
F(U) & =1-\frac{2}{5} \phi^{2}(0)\left(\mathrm{D}_{i}^{2} h_{i}^{2}+\mathrm{D}_{j}^{2} h_{j}^{2}\right) \\
& -\frac{2}{5} \sin ^{2}[\phi(0)] \frac{\phi^{2}(0)}{\tilde{g}_{i j}^{2}(0)}\left(h_{i}-h_{j}\right)^{2}+\mathcal{O}\left(h^{3}\right),
\end{aligned}
$$

where $\mathrm{D}_{l}=\left(\Delta / \Delta_{c}^{l} \Delta_{L}^{l}-1 / \Delta\right) / 2, l=i, j$, are the firstorder coefficients in the Overhauser field in Eq. (17), and the time was expressed through the interaction phase $\phi(0)=\tilde{g}_{i j}(0) t$.

Averaging of the fidelity (18) over both Overhauser fields, we find

$$
\begin{aligned}
\langle F(U)\rangle_{h_{i}, h_{j}}= & 1-\frac{2}{5} \phi^{2}(0) \sigma^{2}\left(\mathrm{D}_{i}^{2}+\mathrm{D}_{j}^{2}\right) \\
& -\frac{4}{5} \frac{\phi^{2}(0)}{\tilde{g}_{i j}^{2}(0)} \sin ^{2}[\phi(0)] \sigma^{2}+\mathcal{O}\left(h^{3}\right) .
\end{aligned}
$$

Assuming the parameters $\Delta_{L}=4.5 \mathrm{meV}, \Delta_{c}=5 \mathrm{meV}$, $g_{c}=0.5 \mathrm{meV}, \Omega_{L}^{i}=\Omega_{L}^{j}=1 \mathrm{meV}$ and $\Delta=0.5 \mathrm{meV}$, such that $\mathrm{D} \equiv \mathrm{D}_{i}=\mathrm{D}_{j}=-0.99 \times 10^{3} \mathrm{eV}^{-1}$ and $\tilde{g}_{i j}=0.02$ $\mathrm{meV}$, the average fidelity given by Eq. (19) behaves as function of $\phi(0)$ as shown in Fig. 4. The value of $\langle F(U)\rangle_{h}$ by $\phi(0)=\pi / 4$ is equal to 0.99995 .

As we can see from the formula 19 , the two effects induced by nuclear spins decrease the fidelity with corresponding strengths: the changing of the electron spinspin coupling strength and resonance conditions $\propto \mathrm{D}^{2}$ and the precession of the rotation axis of the two-electron spin state $\propto 1 / \tilde{g}_{i j}^{2}(0)$. These are determined in Eqs. (5), (16), and (17) and depend on tunable parameters such as $\Delta$ and $\Delta_{L}$ (we can express $\Delta_{c}=\Delta_{L}+\Delta$ ). The cavity detuning can be tuned accordingly, e.g. in photonic two-dimentional slab microcavities using Xe condensation [19]. The contributions to fidelity reduction from terms $\propto \mathrm{D}^{2}$ and $\propto 1 / \tilde{g}_{i j}^{2}(0)$ are not of the same order (s. Fig. 5 b), $\mathrm{D}^{2} \leq 0.1 / \tilde{g}_{i j}^{2}(0)$. But we still keep the terms $\propto \mathrm{D}^{2}$ in our analytical calculations, because they give the upper boundary of the fidelity, when $\sin [\phi(0)]=0$. By changing the laser detuning $\Delta_{L}$ and the detuning $\Delta$ we can adjust the values D and $\tilde{g}_{i j}$ and thus tune the fidelity itself.

The dependence of the fidelity (for $\phi(0)=\frac{\pi}{4}$ ) in respect to $\Delta$ and $\Delta_{L}$ is shown in Fig. 5(a). It indicates that fidelity can be improved considerably by changing the detunings. However by choosing better parameters, some assumptions of the applied theory should be conserved, so that $\tilde{g}_{i j} \ll \Delta$ and $\Delta_{L}\left(\Delta_{c}\right) \gg g_{c}$. One can tune the intensity of applied control laser and rotate the electron spins faster, so that they interact faster as well and "feel" the dephasing in a lesser extent. However the coupling strength $\tilde{g}_{i j}(t)$ depends quadratically on laser intensity and just slight enhancement of Rabi frequency $\Omega_{L}$ will make it of the same order as $\Delta$. Therefore, to improve the fidelity and to keep these relations true one can use e. g. $\Delta=0.3 \mathrm{meV}$ and $\Delta_{L}=4 \mathrm{meV}$. With this choise, the average fidelity for the gate $U\left(\frac{\pi}{4}\right)$ is increased to 0.999991 . The fidelity decays considerably slower in this case as well (Fig. 4). The formula Eq. (15) can be applied for calculating the average fidelity of the CNOT operation. If we neglect the imperfections of the single qubit operations in the CNOT operator sequence and focus only on the decoherence due to the interaction part, then we find for the trace distance between a perfect CNOT operator and CNOT operator with nuclear spins,

$$
\begin{aligned}
& \operatorname{Tr}\left[U_{\mathrm{CNOT}}^{\dagger} U_{\mathrm{CNOT}}(h)\right]= \\
& \left.\quad 2\left(1+\cos \{2[\phi-\phi(0)]\} \cos \left[\left(h_{i}-h_{j}\right) \frac{\phi(0)}{\tilde{g}_{i j}(0)}\right]\right)\right|_{\phi(0)=\frac{\pi}{4}} .
\end{aligned}
$$

The CNOT average fidelity in second order in the Overhauser field is then

$$
\begin{aligned}
F^{\mathrm{CNOT}}= & 1-\frac{16}{5}\left[\phi(0)=\frac{\pi}{4}\right]^{2} \mathrm{D}^{2} \sigma^{2} \\
& -\frac{4}{5}\left[\phi(0)=\frac{\pi}{4}\right]^{2} \frac{\sigma^{2}}{\tilde{g}_{i j}^{2}(0)}+\mathcal{O}\left(h^{3}\right) .
\end{aligned}
$$




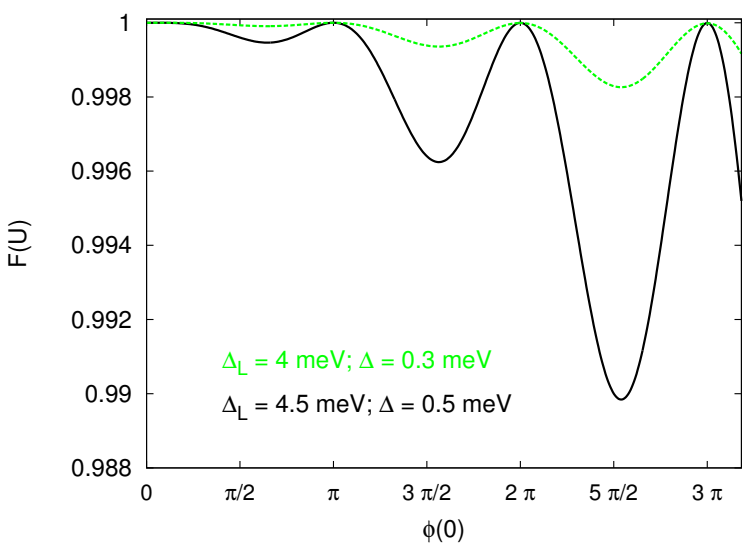

Figure 4. Fidelity $F$ of the unitary time evolution operator $U$ generated by the XY interaction in the presence of nuclear spins as a function of the interaction phase $\phi(0) \propto t$, where $t$ denotes the interaction time. Both lines show the analytical result calculated in second order perturbation theory in the nuclear field. The fidelity can be increased by tuning the parameters $\Delta$ and $\Delta_{L}$. For smaller $\Delta$ and $\Delta_{L}$, the fidelity increases due to faster gate operation. The other parameters are held fixed and are given in the text.

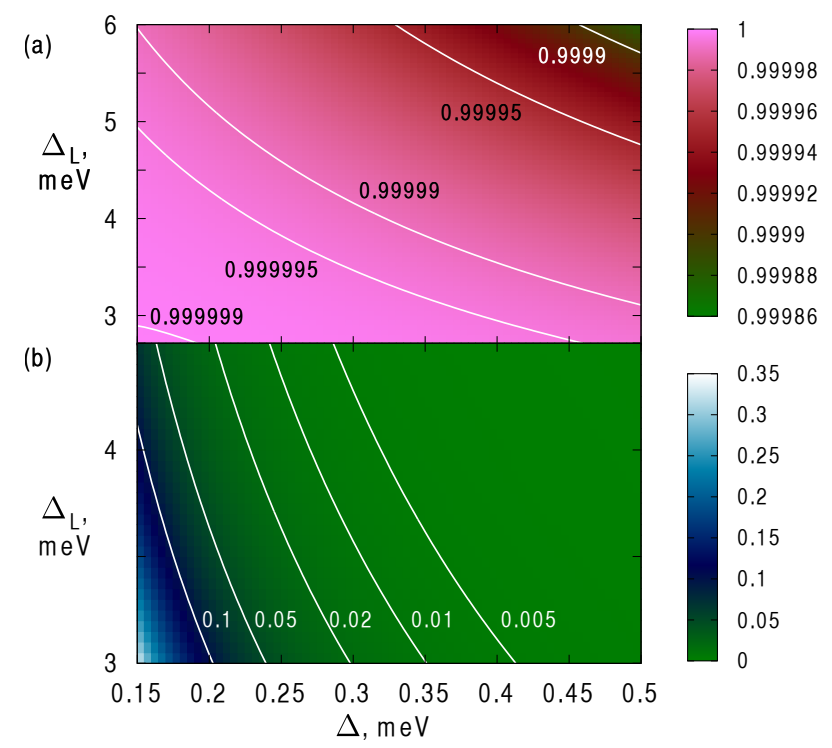

Figure 5. a) The average fidelity $F$ of the unitary time evolution operator $U$ for $\phi(0)=\pi / 4$ calculated in second order of the nuclear field as function of the laser detuning $\Delta_{L}$ and twophoton detuning $\Delta$. An adjustment of these parameters can lead to an increase of the fidelity or to its reduction. However, the choice of the optimal parameters is limited by the conditions of the applied formalism. Further details are given in the text b) The ratio between two contributing mechanism to reduction of the fidelity $\mathrm{D}^{2}\left(\Delta, \Delta_{L}\right) \cdot \tilde{g}_{i j}^{2}\left(\Delta, \Delta_{L}\right)$. It increases for smaller detunings and consequently for improved fidelities.

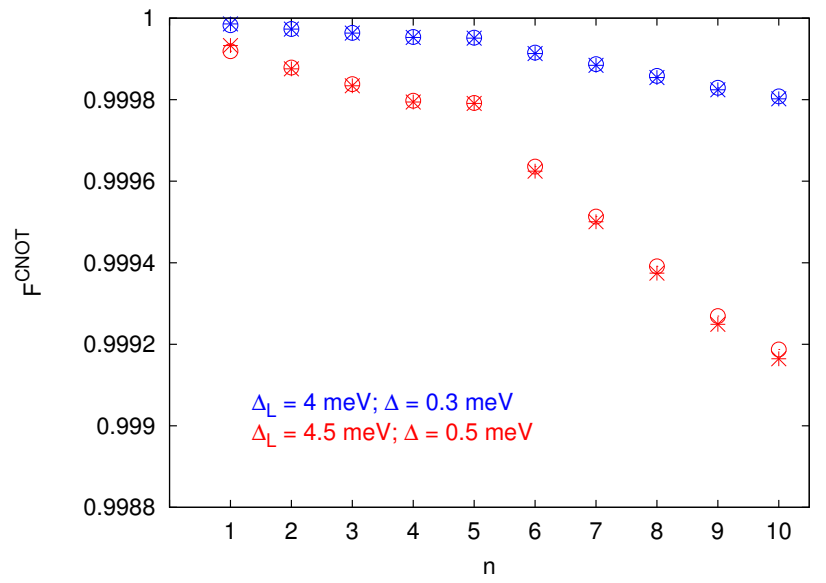

Figure 6. The analytical and numerical results for fidelity of $n$ subsequent CNOT operations in the presence of nuclear spins for different detunings. The analytically calculated values are represented by circles and the numerically calculated ones by stars.

We find that $F^{\mathrm{CNOT}}=0.9999$ by using $\Delta=0.5 \mathrm{meV}$ and $\Delta_{L}=4.5 \mathrm{meV}$, and $F^{\mathrm{CNOT}}=0.999983$ with $\Delta=$ $0.3 \mathrm{meV}$ and $\Delta_{L}=4 \mathrm{meV}$, which is smaller than the fidelity of a single $U\left(\frac{\pi}{4}\right)$ operation.

For the average fidelity for further applied CNOT operations, we find

$$
F^{\mathrm{CNOT}}(n)=\frac{4+\left|\operatorname{Tr}\left\{\left(U_{\mathrm{CNOT}}^{\dagger}\right)^{n}\left[U_{C N O T}(h)\right]^{n}\right\}\right|^{2}}{20} .
$$

The fidelity $F^{\mathrm{CNOT}}(n)$ averaged over the nuclear field (Fig. 6) is reduced after each application and can be increased by a better choice of the detuning parameters. Thus for the last set of parameters only after seven CNOTs, the fidelity drops below typical error correction thresholds $(<0.9999)$ 20].

\section{NUMERICALLY EXACT FIDELITIES}

For large values of the parameter $\phi(0)$ we evaluate the averaging of the fidelities over nuclear fields without expanding $\left|\operatorname{Tr}\left(U(0)^{\dagger} U(h)\right)\right|^{2}$. The results of the numerical averaging are shown in the Fig. 7. The comparison to the analytical result in Fig. 7(a) reveals that our analytical model can predict the behavior of the fidelity only up to a certain value of $\phi(0)$. From Fig. 7)(a) one can see that around $\phi(0) \simeq 12$ the numerical and analytical data begin to differ. For large $\phi(0)$, the analytical data even diverges into negative values. The numerical data on the other hand converges to $1 / 2$. The numerical evaluation of the fidelity for different sets of detunings [Fig. 7 (b)] confirms that the tuning of the transition parameters can improve the fidelity significantly. The numerical curve oscillates with the same period $2 \pi$ as the analytical one. The maxima correspond to the situation, when 

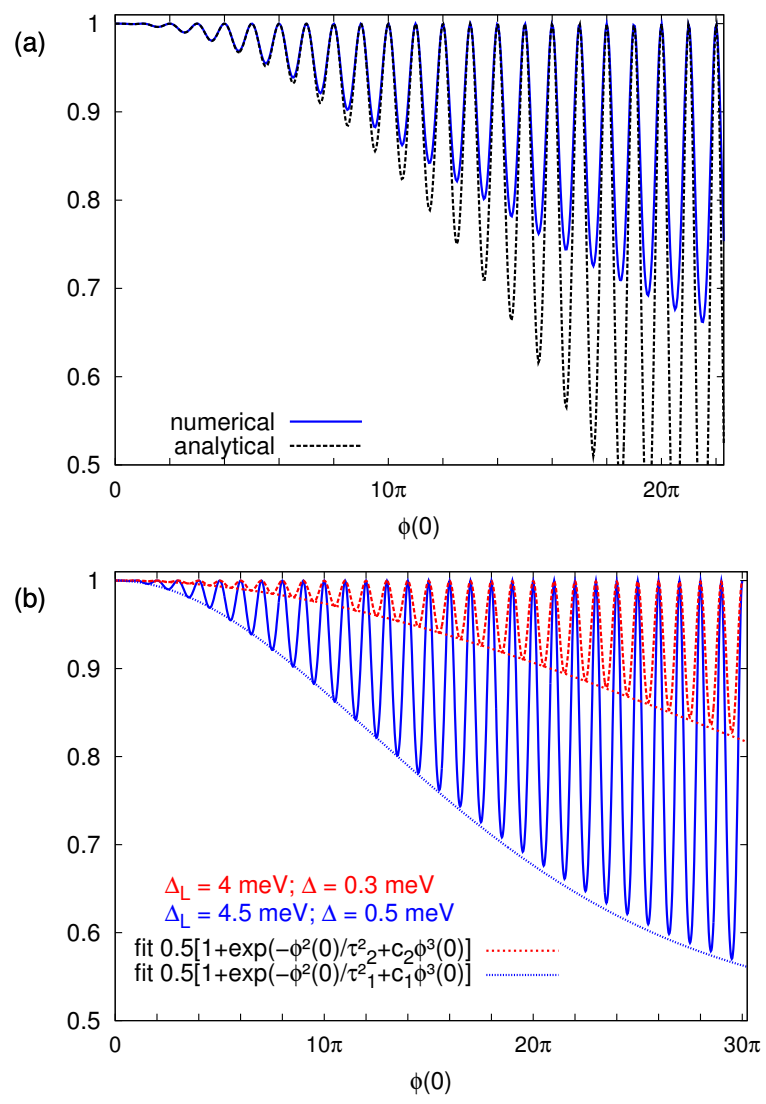

Figure 7. Numerically calculated average fidelity of the time evolution operator for large $\phi(0)$. (a) The comparison between analytical and numerical calculations for $\Delta_{L}=4.5 \mathrm{meV}$ and $\Delta=0.3 \mathrm{meV}$. The analytically obtained fidelity diverges for large $\phi(0)$ while the numerical fidelity saturates to 0.5 . (b) Comparison between two numerically calculated fidelities for the gate $U(\phi)$ with different detunings.

the fidelity is decreased only due to the modified interaction phase between the electron spin and there is no effect of the changed rotation axes of the two-electron spin state. The maxima correspond to the decay of the fidelity, where both Overhauser field induced effects are contributing and the effect of the precession of the rotation axis is maximal.

The fidelity curve suggests that the behavior of its lower boundary could be described by the Gaussian $\sim \mathrm{e}^{-\phi(0)^{2}}$. The fidelity envelope to the second order of $\phi(0)$ is obtained from Eq. 19 by setting $\sin (\phi(0))^{2}$ $=1$, We fit this envelope to the decay function $\frac{1}{2}[1+$ $\left.\exp \left(-\phi(0)^{2} / \tau^{2}\right)\right]$, where $\tau$ is given through parameters $\sigma, \mathrm{D}$ and $\tilde{g}_{i j}$ from Eq. 19 and is defined as

$$
\tau=\sqrt{\frac{5}{8}} \frac{\tilde{g}_{i j}(0)}{\sigma \sqrt{1+\mathrm{D}^{2} \tilde{g}_{i j}^{2}(0)}}=\tau\left(\Delta, \Delta_{L}\right) .
$$

The Gaussian function gives a good fit to the minimum points of the fidelity for small $\phi(0)$, but decays faster for larger $\phi(0)$. The tail of the fidelity can be fitted by adding a third-order term to the argument of the exponential function, $\frac{1}{2}\left[1+\exp \left(-\phi(0)^{2} / \tau^{2}+c \phi(0)^{3}\right)\right]$ with $c$ as a fitting parameter. The fits are shown in Fig. (7) and fit with $\tau_{1}$ and $c_{1}$ corresponds to the curve with parameters $\Delta_{L}=4.5 \mathrm{meV}$ and with $\tau_{2}$ and $c_{2}$ to numerical curve with another set of parameters. The fits yield $c_{1}=9.6 \times 10^{-7}$ and $c_{2}=5 \times 10^{-8}$.

The time dependance of the fidelity can be found by changing from interaction phase $\phi(0)$ to time by assuming $\phi(0)=\tilde{g}_{i j}(0) t$. Then for short time dependance of the lower boundary of the fidelity is described by the function $0.5\left[1+\exp \left(-t^{2} / T^{2}\right)\right]$, where

$$
T=\sqrt{\frac{5}{8}} \frac{\hbar}{\sigma \sqrt{1+\mathrm{D}^{2} \tilde{g}_{i j}^{2}(0)}} .
$$

Since from Fig. (5) we know that $\mathrm{D}^{2} \tilde{g}_{i j}^{2}(0) \ll 1$, we can neglect it here. It follows that $T=\sqrt{\frac{5}{8}} \frac{\hbar}{\sigma}=\sqrt{\frac{5}{8}} \frac{\hbar \sqrt{N}}{A}$, where $N$ is the number of the nuclear spins and $A$ is hyperfine interaction constant. This implies that the decay of the fidelity does not dependend on the interaction strength of the electron spins and is only affected by the nuclear field distributions. By tuning the transition parameters we increase the interaction strength between the coupled electron spins to some reasonable value. Thus the spins acquire a larger interaction phase within the same time. But the decay time of the time evolution operator fidelity is the same and for GaAs $\left(N=10^{5}, A=\right.$ $90 \mu \mathrm{eV}) T=2 \mathrm{~ns}$. The $T_{2}$ decay time of a electron spin coupled to the nuclear bath is given by $T_{2}=\frac{2 \hbar \sqrt{N}}{A}$.21 and in GaAs is $\approx 5$ ns. Since both interacting spins are affected by the decoherence, the decay of the common interaction phase is faster than of a single one $\left(T \approx 0.4 T_{2}\right)$.

The numerically exact CNOT fidelities are presented in Fig. 6. The second order approximation in Overhauser field gives a good agreement to the numerical results. Since the CNOT operator is defined for a fixed interaction phase, there is no oscillation behavior and the fidelity decreases for each further applied CNOT gates.

\section{CONCLUSIONS}

We calculated average fidelities for unitary time evolution operator and CNOT operation of two quantum dots interacting through an off-resonant cavity mode. We obtained results to second order of hyperfine interaction analytically and to an arbitrary order by averaging the fidelities over the Overhauser field distributions numerically. Both approaches are in good agreement for small interaction time durations. If only the longitudinal component of the Overhauser field in two interacting quantum dots is considered for the hyperfine interaction, then the time evolution of the two-electron spin state changes in two ways (Fig. 3), and both effects contribute differently to the reduction of the fidelity. A prolongation of 
the decoherence time of the two-electron state is possible by narrowing the distribution of the Overhauser field [22] [Eq. [19], (21)]. The precession of the rotion axis of the two-electron state on the Bloch sphere can be minimized by preparing the the nuclear spin states in each interacting quantum dot in the same certain state and minimizing the fluctuations of the Overhauser fields [23]. Since we neglected the hyperfine spin-flip terms in our calculations, the obtained results are only valid for the Zeeman fields $\propto 1 \mathrm{~T}$.

\section{ACKNOWLEDGMENTS}

We acknowledge funding from the DFG within SFB 767.
[1] M. H. Mikkelsen, J. Berezovsky, N. G. Stoltz, L. A. Coldren, and D. D. Awschalom, Nature Phys. 3, 770 (2007).

[2] J. Berezovsky, M. H. Mikkelsen, N. G. Stoltz, L. Coldren, and D. D. Awschalom, Science 320, 349 (2008).

[3] S. Yilmaz, P. Fallahi, and A. Imamoglu, Phys. Rev. Let. 105, 033601 (2010).

[4] R.-B. Liu, W. Yao, and L. J. Sham, Advances in Physics 59, 69 (2010).

[5] D. Loss and D. P. DiVincenzo, Phys. Rev. A 57, 120 (1998).

[6] A. V. Khaetskii, D. Loss, and L. Glazman, Phys. Rev. Lett. 88, 186802 (2002).

[7] W. A. Coish and J. Baugh, Phys. Stat. Solidi (b) 246, 2203 (2009).

[8] J. R. Petta, A. C. Johnson, J. M. Taylor, E. A. Laird, A. Yacoby, M. D. Lukin, C. M. Marcus, M. P. Hanson, and A. C. Gossard, Science 309, 2180 (2005).

[9] F. H. L. Koppens, C. Buizert, K. J. Tielrooij, I. T. Vink, K. C. Nowack, T. Meunier, L. P. Kouwenhoven, and L. M. K. Vandersypen, Nature 442, 766 (2006).

[10] H. Bluhm, S. Foletti, I. Neder, M. Rudner, D. Mahalu, V. Umansky, and A. Yacoby, Nat. Phys. 7, 109 (2011).

[11] S. Foletti, H. Bluhm, D. Mahalu, V. Umansky, and A. Yacoby, Nature Phys. 5, 903 (2009)
[12] B. Trauzettel, D.V. Bulaev, D. Loss, and G. Burkard, Nature Phys. 3, 192 (2007).

[13] R. Hanson and D. D. Awschalom, Nature 453, 1043 (2006).

[14] H. Ribeiro, J. R. Petta, G. Burkard, Phys. Rev. B 82, 115445 (2010).

[15] A. Imamoglu, D. D. Awschalom, G. Burkard, D. P. DiVincenzo, D. Loss, M. Sherwin, and A. Small, Phys. Rev. Lett. 83, 4204 (1999).

[16] V. Cerletti, W. A. Coish, O. Gywat, and D. Loss, Nanotechnology 16, R27 (2005).

[17] L. H. Petersen, N. M. Møller, K. Mølmer, Phys. Lett. A 367, 47 (2007).

[18] J. Ghosh and M. R. Geller, Phys. Rev. A 81, 052340 (2010).

[19] G. Khitrova, H. M. Gibbs, M. Kira, S.W. Koch, and A. Scherer, Nature Phys. 2, 81 (2006).

[20] M. A Nielsen and I. L. Chuang, Quantum Computation and Quantum Information (Cambridge University Press, Cambridge, UK, 2009).

[21] W. A. Coish and D. Loss, Phys. Rev B, 70, 195340 (2004).

[22] D. Stepanenko, G. Burkard, G. Giedke, and A. Imamoglu, Phys. Rev. Lett. 96, 136401 (2006).

[23] H. Ribeiro and G. Burkard, Phys. Rev. Lett. 102, 216802 (2009). 